\title{
Fundamental Group and Covering Properties of Hyperbolic Surgery Manifolds
}

\author{
Alberto Cavicchioli, Fulvia Spaggiari, and Agnese Ilaria Telloni \\ Dipartimento di Scienze Fisiche, Informatiche e Matematiche, Università di Modena e Reggio E., Via Campi 213/B, \\ 41100 Modena, Italy
}

Correspondence should be addressed to Alberto Cavicchioli; alberto.cavicchioli@unimore.it

Received 10 June 2013; Accepted 27 August 2013

Academic Editor: Manuel Sanchis

Copyright ( 2013 Alberto Cavicchioli et al. This is an open access article distributed under the Creative Commons Attribution License, which permits unrestricted use, distribution, and reproduction in any medium, provided the original work is properly cited.

\begin{abstract}
We study a family of closed connected orientable 3-manifolds obtained by Dehn surgeries with rational coefficients along the oriented components of certain links. This family contains all the manifolds obtained by surgery along the (hyperbolic) 2-bridge knots. We find geometric presentations for the fundamental group of such manifolds and represent them as branched covering spaces. As a consequence, we prove that the surgery manifolds, arising from the hyperbolic 2-bridge knots, have Heegaard genus 2 and are 2 -fold coverings of the 3 -sphere branched over well-specified links.
\end{abstract}

\section{Manifolds Obtained by Dehn Surgeries}

As well known, any closed connected orientable 3-manifold can be obtained by Dehn surgeries on the components of an oriented link in the 3 -sphere (see $[1,2])$. If such a link is hyperbolic, then the Thurston-Jorgensen theory [3] of hyperbolic surgery implies that the resulting manifolds are hyperbolic for almost all surgery coefficients. Another method for studying a closed orientable 3-manifold is to represent it as a branched covering of a link in the 3-sphere (see, e.g., [4]). If such a link is hyperbolic, then the construction yields hyperbolic manifolds for branching indices sufficiently large. In the context of current research in 3-manifold topology, many classes of closed orientable hyperbolic 3-manifolds have been constructed by considering branched coverings of links or by performing Dehn surgery along them (see, e.g., [5-10]). This paper relates these methods to study a new class of hyperbolic orientable 3-manifolds via combinatorial tools. More precisely, for any positive integer $n$, let $\mathscr{L}_{2 n+1}$ be the oriented link with $2 n+1$ components $L_{0}, L_{i}$, and $K_{i}, i=$ $1, \ldots, n$, in the oriented 3-sphere $\mathbb{S}^{3}$ depicted in Figure 1. This link can be obtained as a belted sum of Borromean rings, as remarked in [11, p. 8]; thus, it is hyperbolic for any $n \geq 1$. Let us consider the closed connected orientable 3-manifolds
$M_{n}\left(r_{i} / s_{i} ; p_{i} / q_{i} ; h / k\right)$ obtained by Dehn surgery on $\mathbb{S}^{3}$ along the oriented link $\mathscr{L}_{2 n+1}$ such that the surgery coefficients $r_{i} / s_{i}$, $p_{i} / q_{i}$, and $h / k$ correspond to the oriented components $L_{i}, K_{i}$, and $L_{0}$, respectively, where $i=1, \ldots, n$. Of course, we always assume that $\operatorname{gcd}\left(r_{i}, s_{i}\right)=1, \operatorname{gcd}\left(p_{i}, q_{i}\right)=1$, and $\operatorname{gcd}(h, k)=1$. Here we will show that our family of manifolds contains all closed manifolds obtained by Dehn surgeries on 2-bridge knots. Such manifolds and their geometries were studied in a nice paper of Brittenham and $\mathrm{Wu}$, where the exceptional Dehn surgeries on 2-bridge knots were completely classified (see [5]). This fact gives a further motivation for the study of our surgery manifolds. Recall that a nontrivial Dehn surgery on a hyperbolic knot in the oriented 3-sphere is said to be exceptional if the resulting manifold is either reducible, toroidal, or a Seifert fibered manifold whose orbifold base is the 2-sphere with at most three exceptional fibers (called a small Seifert fibered space). Thus an exceptional Dehn surgery is not hyperbolic. Moreover, it can be shown that a nonexceptional surgery on a 2-bridge knot is hyperbolic (see [5]). Now we determine a geometric presentation for the fundamental group of the surgery manifold $M_{n}\left(r_{i} / s_{i} ; p_{i} / q_{i} ; h / k\right)$. A group presentation is said to be geometric if it arises from a Heegaard diagram of a closed connected (orientable) 3-manifold. If so, then the presentation also corresponds to a spine of the 


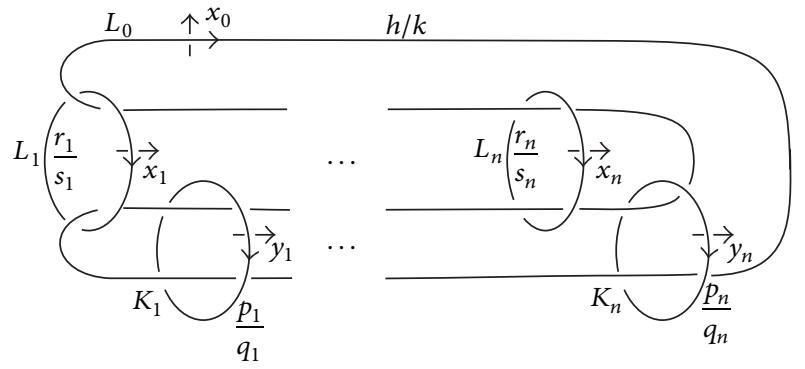

Figure 1: Dehn surgery description of the 3-manifold $M_{n}\left(r_{i} / s_{i} ; p_{i} / q_{i} ; h / k\right)$ and the generators of a Wirtinger presentation of $\pi\left(\mathscr{L}_{2 n+1}\right)$.

considered manifold. A Wirtinger presentation of the link group $\pi\left(\mathscr{L}_{2 n+1}\right)=\pi_{1}\left(\mathbb{S}^{3} \backslash \mathscr{L}_{2 n+1}\right)$ has generators $x_{0}, x_{i}$, and $y_{i}$, for every $i=1, \ldots, n$ (see Figure 1 ).

The meridians $\mathbf{m}_{i}$ and $\boldsymbol{\mu}_{i}$ and the longitudes $\boldsymbol{\ell}_{i}$ and $\boldsymbol{\lambda}_{i}$ of the components $L_{i}$ and $K_{i}$, respectively, of $\mathscr{L}_{2 n+1}$ are

$$
\begin{gathered}
\mathbf{m}_{i}=x_{i}, \quad \boldsymbol{e}_{i}=y_{i-1}^{-1} x_{i-1}^{-1} \cdots y_{1}^{-1} x_{1}^{-1} y_{1} \cdots y_{n} x_{0} y_{n}^{-1} \cdots y_{1}^{-1} \\
x_{1} y_{1} \cdots x_{i-1} y_{i-1} x_{i-1}^{-1} \cdots x_{1}^{-1} x_{0}^{-1} x_{1} \cdots x_{i-1}, \\
\boldsymbol{\mu}_{i}=y_{i}, \quad \boldsymbol{\lambda}_{i}=y_{i} \cdots y_{n} x_{0}^{-1} y_{n}^{-1} \cdots y_{i}^{-1} x_{i}^{-1} y_{i-1}^{-1} \cdots x_{2}^{-1} y_{1}^{-1} x_{1}^{-1} \\
\\
y_{1} \cdots y_{n} x_{0} y_{n}^{-1} \cdots y_{1}^{-1} x_{1} y_{1} x_{2} \cdots y_{i-1} x_{i},
\end{gathered}
$$

where $\left[\mathbf{m}_{i}, \boldsymbol{\ell}_{i}\right]=1$ and $\left[\boldsymbol{\mu}_{i}, \boldsymbol{\lambda}_{i}\right]=1$ for every $i=1, \ldots, n$. The meridian $\mathbf{m}_{0}$ and the longitude $\boldsymbol{\ell}_{0}$ of the component $L_{0}$ of $\mathscr{L}_{2 n+1}$ are

$$
\mathbf{m}_{0}=x_{0}, \quad \boldsymbol{e}_{0}=y_{n}^{-1} \cdots y_{1}^{-1} x_{1} y_{1} \cdots x_{n} y_{n} x_{n}^{-1} \cdots x_{1}^{-1} .
$$

To determine the formulae for longitudes $\boldsymbol{\ell}_{i}, \boldsymbol{\lambda}_{i}$, and $\boldsymbol{\ell}_{0}$, we have used the following procedure. Fix an orientation and an initial point for each component of the link $\mathscr{L}_{2 n+1}$. Starting from the initial point, we run along the component in the sense of the fixed orientation and write in order only the generators encountered at the undercrossings. At each undercrossing we write the generator (represented by the oriented arc running over the undercrossing) with positive (resp., negative) exponent if the sense of percorrence is equal (resp., opposite) to the orientation of the named arc. The obtained longitude is homologous to zero in the complement of the considered component if the exponent sum is equal to zero.

A finite presentation for the fundamental group of the surgery manifold $M_{n}\left(r_{i} / s_{i} ; p_{i} / q_{i} ; h / k\right)$ is obtained from that of $\pi\left(\mathscr{L}_{2 n+1}\right)$ by adding the relations

$$
\mathbf{m}_{i}^{r_{i}} \boldsymbol{\ell}_{i}^{s_{i}}=1, \quad \boldsymbol{\mu}_{i}^{p_{i}} \boldsymbol{\lambda}_{i}^{q_{i}}=1, \quad \mathbf{m}_{0}^{h} \boldsymbol{\ell}_{0}^{k}=1,
$$

for $i=1, \ldots, n$. Since the integers of the pairs $\left(p_{i}, q_{i}\right),\left(r_{i}, s_{i}\right)$, and $(h, k)$ are coprime, there are integers $\alpha_{i}, \beta_{i}, \gamma_{i}, \delta_{i}, \xi$, and $\eta$ such that

$$
\begin{gathered}
q_{i} \alpha_{i}-p_{i} \beta_{i}=1, \\
s_{i} \gamma_{i}-r_{i} \delta_{i}=1, \\
k \xi-h \eta=1 .
\end{gathered}
$$

Let us define

$$
\begin{aligned}
a_{i} & :=\mathbf{m}_{i}^{\gamma_{i}} \boldsymbol{\ell}_{i}^{\delta_{i}}, \\
b_{i} & :=\boldsymbol{\mu}_{i}^{\alpha_{i}} \lambda_{i}^{\beta_{i}}, \\
c & :=\mathbf{m}_{0}^{\xi} \boldsymbol{\ell}_{0}^{\eta},
\end{aligned}
$$

for $i=1, \ldots, n$.

Then we have

$$
\begin{aligned}
& a_{i}^{s_{i}}=\left(\mathbf{m}_{i}^{\gamma_{i}} \boldsymbol{e}_{i}^{\delta_{i}}\right)^{s_{i}}=\mathbf{m}_{i} \mathbf{m}_{i}^{r_{i} \delta_{i}} \boldsymbol{\ell}_{i}^{\delta_{i} s_{i}}=\mathbf{m}_{i}\left(\mathbf{m}_{i}^{r_{i}} \boldsymbol{e}_{i}^{s_{i}}\right)^{\delta_{i}}=\mathbf{m}_{i}=x_{i}, \\
& b_{i}^{q_{i}}=\left(\boldsymbol{\mu}_{i}^{\alpha_{i}} \lambda_{i}^{\beta_{i}}\right)^{q_{i}}=\boldsymbol{\mu}_{i} \boldsymbol{\mu}_{i}^{p_{i} \beta_{i}} \lambda_{i}^{q_{i} \beta_{i}}=\boldsymbol{\mu}_{i}\left(\boldsymbol{\mu}_{i}^{p_{i}} \lambda_{i}^{q_{i}}\right)^{\beta_{i}}=\boldsymbol{\mu}_{i}=y_{i}, \\
& c^{k}=\left(\mathbf{m}_{0}^{\xi} \boldsymbol{\ell}_{0}^{\eta}\right)^{k}=\mathbf{m}_{0} \mathbf{m}_{0}^{h \eta} \boldsymbol{\ell}_{0}^{k \eta}=\mathbf{m}_{0}\left(\mathbf{m}_{0}^{h} \boldsymbol{\ell}_{0}^{k}\right)^{\eta}=\mathbf{m}_{0}=x_{0}, \\
& a_{i}^{-r_{i}}=\left(\mathbf{m}_{i}^{\gamma_{i}} \boldsymbol{\ell}_{i}^{\delta_{i}}\right)^{-r_{i}}=\mathbf{m}_{i}^{-r_{i} \gamma_{i}} \boldsymbol{\ell}_{i}^{-s_{i} \gamma_{i}} \boldsymbol{\ell}_{i}=\left(\mathbf{m}_{i}^{r_{i}} \boldsymbol{\ell}_{i}^{s_{i}}\right)^{-\gamma_{i}} \boldsymbol{\ell}_{i}=\boldsymbol{\ell}_{i}, \\
& b_{i}^{-p_{i}}=\left(\boldsymbol{\mu}_{i}^{\alpha_{i}} \lambda_{i}^{\beta_{i}}\right)^{-p_{i}}=\boldsymbol{\mu}_{i}^{-p_{i} \alpha_{i}} \lambda_{i}^{-q_{i} \alpha_{i}} \boldsymbol{\lambda}_{i}=\left(\boldsymbol{\mu}_{i}^{p_{i}} \lambda_{i}^{q_{i}}\right)^{-\alpha_{i}} \boldsymbol{\lambda}_{i}=\lambda_{i}, \\
& c^{-h}=\left(\mathbf{m}_{0}^{\xi} \boldsymbol{\ell}_{0}^{\eta}\right)^{-h}=\mathbf{m}_{0}^{-h \xi} \boldsymbol{\ell}_{0}^{-k \xi} \boldsymbol{\ell}_{0}=\left(\mathbf{m}_{0}^{h} \boldsymbol{\ell}_{0}^{k}\right)^{-\xi} \boldsymbol{\ell}_{0}=\boldsymbol{\ell}_{0}
\end{aligned}
$$

for $i=1, \ldots, n$. We have the following result.

Theorem 1. The fundamental group of the surgery 3-dimensional manifold $M_{n}\left(r_{i} / s_{i} ; p_{i} / q_{i} ; h / k\right)$ admits the finite balanced presentation with $2 n+1$ generators $a_{i}, b_{i}$, and $c, i=1, \ldots, n$, and $2 n+1$ relations:

$$
\begin{gathered}
a_{i}^{r_{i}} b_{i}^{q_{i}} \cdots b_{n}^{q_{n}} c^{k} b_{n}^{-q_{n}} \cdots b_{i}^{-q_{i}} c^{-k}=1, \\
b_{i}^{p_{i}} c^{-k} a_{i}^{-s_{i}} \cdots a_{1}^{-s_{1}} c^{k} a_{1}^{s_{1}} \cdots a_{i}^{s_{i}}=1, \\
c^{h} b_{n}^{-q_{n}} \cdots b_{1}^{-q_{1}} a_{1}^{s_{1}} b_{1}^{q_{1}} \cdots a_{n}^{s_{n}} b_{n}^{q_{n}} a_{n}^{-s_{n}} \cdots a_{1}^{-s_{1}}=1 .
\end{gathered}
$$

The closed manifold $M_{n}\left(r_{i} / s_{i} ; p_{i} / q_{i} ; h / k\right)$ admits a Heegaard diagram of genus $2 n+1$ inducing the above presentation, which is thus geometric. Furthermore, the Heegaard genus of $M_{n}\left(r_{i} / s_{i} ; p_{i} / q_{i} ; h / k\right)$ is at most $2 n+1$.

Proof. Substituting the above relations in the relators of the Wirtinger presentation of $\pi\left(\mathscr{L}_{2 n+1}\right)$ and using the previous formulae for the longitudes $\boldsymbol{\ell}_{i}, \boldsymbol{\lambda}_{i}$, and $\boldsymbol{\ell}_{0}$, we get the relations of the statement. More precisely, substituting $\boldsymbol{\ell}_{1}=a_{1}^{-r_{1}}, y_{i}=$ $b_{i}^{q_{i}}$, and $x_{0}=c^{k}$ into

$$
\boldsymbol{\ell}_{1}=y_{1} \cdots y_{n} x_{0} y_{n}^{-1} \cdots y_{1}^{-1} x_{0}^{-1}
$$


we get

$$
a_{1}^{-r_{1}}=b_{1}^{q_{1}} \cdots b_{n}^{q_{n}} c^{k} b_{n}^{-q_{n}} \cdots b_{1}^{-q_{1}} c^{-k}
$$

or, equivalently,

$$
a_{1}^{r_{1}} b_{1}^{q_{1}} \cdots b_{n}^{q_{n}} c^{k} b_{n}^{-q_{n}} \cdots b_{1}^{-q_{1}} c^{-k}=1
$$

which is the first relation of the statement for $i=1$. Then we have

$$
\begin{aligned}
b_{1}^{-p_{1}}=\lambda_{1}= & \left(y_{1} \cdots y_{n} x_{0}^{-1} y_{n}^{-1} \cdots y_{1}^{-1}\right) x_{1}^{-1} \\
& \times\left(y_{1} \cdots y_{n} x_{0} y_{n}^{-1} \cdots y_{1}^{-1}\right) x_{1} \\
= & \left(\boldsymbol{e}_{1} x_{0}\right)^{-1} x_{1}^{-1}\left(\boldsymbol{e}_{1} x_{0}\right) x_{1} \\
= & c^{-k} a^{r_{1}} a_{1}^{-s_{1}} a_{1}^{-r_{1}} c^{k} a_{1}^{s_{1}} \\
= & c^{-k} a_{1}^{-s_{1}} c^{k} a_{1}^{s_{1}}
\end{aligned}
$$

or, equivalently,

$$
b_{1}^{p_{1}} c^{-k} a_{1}^{-s_{1}} c^{k} a_{1}^{s_{1}}=1
$$

which is the second relation of the statement for $i=1$. From the expression of $\boldsymbol{\ell}_{2}$ we get

$$
\begin{aligned}
a_{2}^{-r_{2}}=\boldsymbol{\ell}_{2} & =y_{1}^{-1} x_{1}^{-1}\left(y_{1} \cdots y_{n} x_{0} y_{n}^{-1} \cdots y_{1}^{-1}\right) x_{1} y_{1} x_{1}^{-1} x_{0}^{-1} x_{1} \\
& =b_{1}^{-q_{1}} a_{1}^{-s_{1}}\left(\boldsymbol{e}_{1} x_{0}\right) a_{1}^{s_{1}} b_{1}^{q_{1}} a_{1}^{-s_{1}} c^{-k} a_{1}^{s_{1}} \\
& =b_{1}^{-q_{1}} a_{1}^{-s_{1}} a_{1}^{-r_{1}} c^{k} a_{1}^{s_{1}} b_{1}^{q_{1}} a_{1}^{-s_{1}} c^{-k} a_{1}^{s_{1}} \\
& =b_{1}^{-q_{1}} a_{1}^{-r_{1}}\left(a_{1}^{-s_{1}} c^{k} a_{1}^{s_{1}}\right) b_{1}^{q_{1}}\left(a_{1}^{-s_{1}} c^{-k} a_{1}^{s_{1}}\right) \\
& =b_{1}^{-q_{1}} a_{1}^{-r_{1}} c^{k} b_{1}^{-p_{1}} b_{1}^{q_{1}} b_{1}^{p_{1}} c^{-k} \\
& =b_{1}^{-q_{1}} a_{1}^{-r_{1}} c^{k} b_{1}^{q_{1}} c^{-k} \\
& =b_{1}^{-q_{1}}\left(b_{1}^{q_{1}} \cdots b_{n}^{q_{n}} c^{k} b_{n}^{-q_{n}} \cdots b_{1}^{-q_{1}} c^{-k}\right) c^{k} b_{1}^{q_{1}} c^{-k} \\
& =b_{2}^{q_{2}} \cdots b_{n}^{q_{n}} c^{k} b_{n}^{-q_{n}} \cdots b_{2}^{-q_{2}} c^{-k}
\end{aligned}
$$

or, equivalently,

$$
a_{2}^{r_{2}} b_{2}^{q_{2}} \cdots b_{n}^{q_{n}} c^{k} b_{n}^{-q_{n}} \cdots b_{2}^{-q_{2}} c^{-k}=1
$$

which is the first relation of the statement for $i=2$. From the expression of $\lambda_{2}$ we get

$$
\begin{aligned}
b_{2}^{-p_{2}}=\lambda_{2}= & \left(y_{2} \cdots y_{n} x_{0}^{-1} y_{n}^{-1} \cdots y_{2}^{-1}\right) x_{2}^{-1} y_{1}^{-1} x_{1}^{-1} \\
& \times\left(y_{1} \cdots y_{n} x_{0} y_{n}^{-1} \cdots y_{1}^{-1}\right) x_{1} y_{1} x_{2} \\
= & \left(b_{2}^{q_{2}} \cdots b_{n}^{q_{n}} c^{-k} b_{n}^{-q_{n}} \cdots b_{2}^{-q_{2}}\right) a_{2}^{-s_{2}} b_{1}^{-q_{1}} a_{1}^{-s_{1}} \\
& \times\left(b_{1}^{q_{1}} \cdots b_{n}^{q_{n}} c^{k} b_{n}^{-q_{n}} \cdots b_{1}^{-q_{1}}\right) a_{1}^{s_{1}} b_{1}^{q_{1}} a_{2}^{s_{2}} \\
= & c^{-k} a_{2}^{r_{2}} a_{2}^{-s_{2}}\left(b_{1}^{-q_{1}} a_{1}^{-s_{1}} a_{1}^{-r_{1}} c^{k} a_{1}^{s_{1}} b_{1}^{q_{1}}\right) a_{2}^{s_{2}} \\
= & c^{-k} a_{2}^{-s_{2}} a_{2}^{r_{2}} a_{2}^{-r_{2}} a_{1}^{-s_{1}} c^{k} a_{1}^{s_{1}} a_{2}^{s_{2}} \\
= & c^{-k} a_{2}^{-s_{2}} a_{1}^{-s_{1}} c^{k} a_{1}^{s_{1}} a_{2}^{s_{2}}
\end{aligned}
$$

or, equivalently,

$$
b_{2}^{p_{2}} c^{-k} a_{2}^{-s_{2}} a_{1}^{-s_{1}} c^{k} a_{1}^{s_{1}} a_{2}^{s_{2}}=1
$$

which is the second relation of the statement for $i=2$. Going on like this, we get by finite iteration the first and second relations of the statement for $i=1, \ldots, n$. Substituting $\boldsymbol{\ell}_{0}=$ $c^{-h}, y_{i}=b_{i}^{q_{i}}$, and $x_{i}=a_{i}^{s_{i}}$ into

$$
\boldsymbol{\ell}_{0}=y_{n}^{-1} \cdots y_{1}^{-1} x_{1} y_{1} \cdots x_{n} y_{n} x_{n}^{-1} \cdots x_{1}^{-1}
$$

we get

$$
c^{-h}=b_{n}^{-q_{n}} \cdots b_{1}^{-q_{1}} a_{1}^{s_{1}} b_{1}^{q_{1}} \cdots a_{n}^{s_{n}} b_{n}^{q_{n}} a_{n}^{-s_{n}} \cdots a_{1}^{-s_{1}}
$$

which gives the last relation of the statement. To show that the presentation in Theorem 1 is geometric, it suffices to draw a suitable RR-system (Rail-Road system) which induces precisely the above presentation (see Figure 2). The hexagons represent the generators, and the three curves labelled by 1 , 2 , or 3 arrows correspond to the relations in the statement of Theorem 1. For the theory of RR-systems we refer the reader to $[12,13]$.

We also note that the first integral homology group of $M_{n}\left(r_{i} / s_{i} ; p_{i} / q_{i} ; h / k\right)$ is isomorphic to $\bigoplus_{i=1}^{n}\left(\mathbb{Z}_{\left|r_{i}\right|} \oplus \mathbb{Z}_{\left|p_{i}\right|}\right) \oplus \mathbb{Z}_{|h|}$. For example, if $r_{i}=p_{i}=h=0, i=1, \ldots, n$, then the Heegaard genus of our surgery manifolds is exactly $2 n+1$.

As remarked in [11, p. 8], the link $\mathscr{L}_{2 n+1}$ is hyperbolic in the sense that it has a hyperbolic complement. So the Thurston-Jorgensen theory [3] of hyperbolic surgery gives the following result.

Theorem 2. For any integer $n \geq 1$ and for almost all pairs of surgery coefficients $r_{i} / s_{i}, p_{i} / q_{i}$, and $h / k$, the closed connected orientable 3-manifolds $M_{n}\left(r_{i} / s_{i} ; p_{i} / q_{i} ; h / k\right)$ are hyperbolic.

If $r_{i}=p_{i}=1$ for every $i=1, \ldots, n$, then the surgery 3 dimensional manifold $M_{n}\left(1 / s_{i} ; 1 / q_{i} ; h / k\right)$ is homeomorphic to the closed orientable 3 -manifold $K_{\alpha / \beta}(h / k)$ obtained by $h / k$ Dehn surgery on the 2 -bridge knot $K_{\alpha / \beta}$ corresponding to the Conway parameters $\left[-2 s_{1}, 2 q_{1}, \ldots,-2 s_{n}, 2 q_{n}\right]$, as shown in Figure 3. Note that our parameterization is coherent with that used by Rolfsen [4, p. 303], by setting $c_{1}=-2 s_{1}, c_{2}=2 q_{1}$, and so on. The $c_{i}$ in Rolfsen notation indicate the number of crossings and are negative if the sense of the crossings is reversed. This implies that our picture in Figure 3 is slightly different to that drawn in Rolfsen [4, p. 303], as $c_{i}$ and $2 s_{i}$ have opposite signs for $i$ odd. In particular, $c_{i}$ is negative for $i$ odd since $s_{i} \geq 1$. We always assume that $k \neq 0$; that is, the surgery on $K_{\alpha / \beta}$ is nontrivial. See [14] for the Conway notation of 2bridge knots. Here $\alpha$ and $\beta$ are coprime integers given by the continued fraction

$$
\frac{\alpha}{\beta}=-2 s_{1}+\frac{1}{2 q_{1}+\cdots+1 /\left(-2 s_{n}+1 / 2 q_{n}\right)},
$$

where $\alpha>0,-\alpha<\beta<\alpha$, and $\alpha$ (resp., $\beta$ ) is odd (resp., even), and $s_{i}, q_{i} \geq 1$ for $i=1, \ldots, n$.

Since every 2 -bridge knot admits a Conway representation with an even number of even parameters (see exercise 


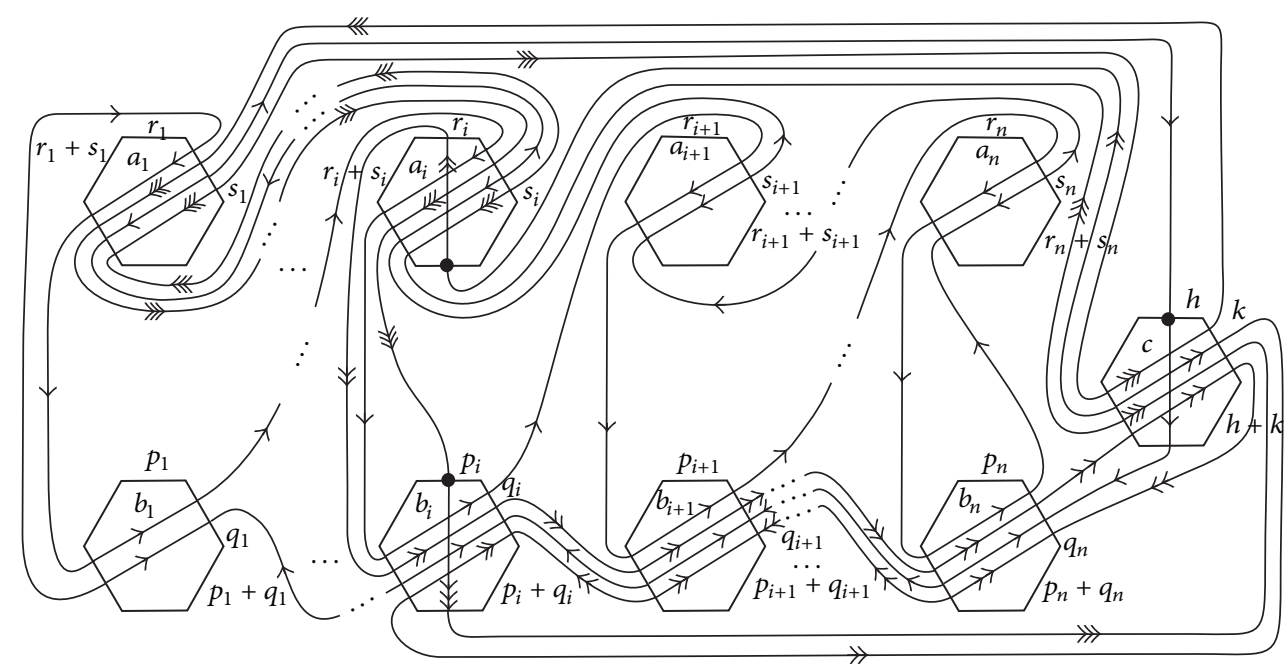

FIGURE 2: An RR-system of genus $2 n+1$ inducing the presentation of $\pi_{1}\left(M_{n}\left(r_{i} / s_{i} ; p_{i} / q_{i} ; h / k\right)\right)$.

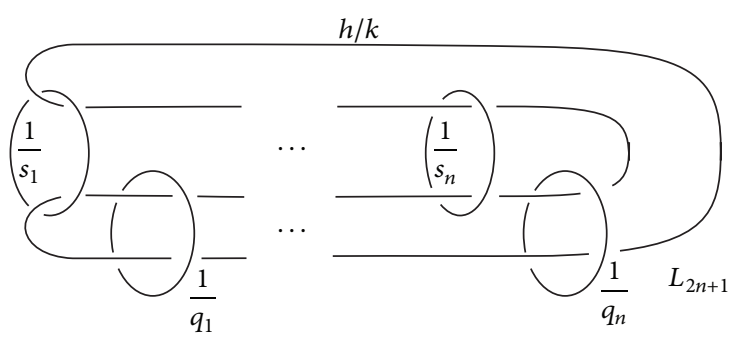

(a)

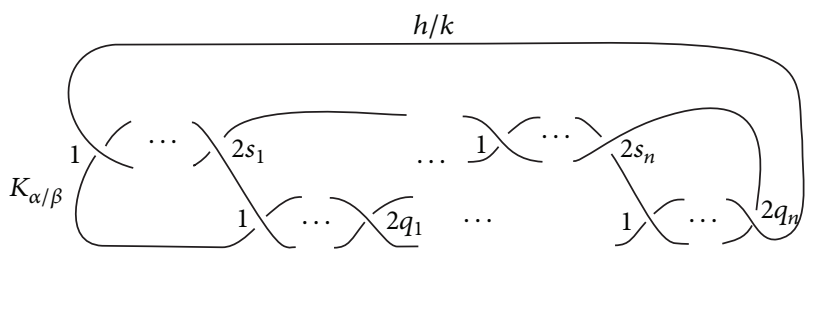

(b)

FIGURE 3: Two equivalent surgery descriptions of the surgery manifold $K_{\alpha / \beta}(h / k)$, where $\alpha / \beta=\left[-2 s_{1}, 2 q_{1}, \ldots,-2 s_{n}, 2 q_{n}\right]$.

2.1.14 of $[15$, p. 26]), we have that our family of surgery manifolds $M_{n}\left(r_{i} / s_{i} ; p_{i} / q_{i} ; h / k\right)$ contains all closed manifolds obtained by (nontrivial) Dehn surgeries on 2-bridge knots. Recall that a 2-bridge knot $K_{\alpha / \beta}$ is nonhyperbolic if and only if $\alpha=1$, in which case it is the torus knot of type $(2, \beta)$ (see, e.g., [5]). Since the surgery on torus knot is well understood (see [9]), we restrict our attention to hyperbolic 2-bridge knots. Ochiai proved that such manifolds have Heegaard genus 2 (see [10]). The following also gives a different proof of the Ochiai result together with an explicit 2-generator 2-relator geometric presentation of the fundamental group.

Theorem 3. Let $K_{\alpha / \beta}(\gamma), \gamma=h / k \neq \infty$, be the closed orientable 3-manifold obtained by $\gamma$ Dehn surgery on the hyperbolic 2bridge knot $K_{\alpha / \beta}$, where $\alpha / \beta=\left[-2 s_{1}, 2 q_{1}, \ldots,-2 s_{n}, 2 q_{n}\right]$. Then the fundamental group of $K_{\alpha / \beta}(\gamma)$ admits a geometric presentation with generators $a_{1}$ and $c$ and two relators deduced from the recurrence formulae:

$$
\begin{gathered}
a_{i+1}=c^{k} b_{i}^{-q_{i}} c^{-k} a_{i} b_{i}^{q_{i}}, \\
b_{i+1}=a_{i+1}^{-s_{i+1}} b_{i} c^{-k} a_{i+1}^{s_{i+1}} c^{k}
\end{gathered}
$$

for $i=1, \ldots, n-1$, where $b_{1}=a_{1}^{-s_{1}} c^{-k} a_{1}^{s_{1}} c^{k}$. In particular, the surgery manifold $K_{\alpha / \beta}(\gamma)$ has Heegaard genus 2 .

Proof. By Theorem 1, the fundamental group of $K_{\alpha / \beta}(\gamma)$ has a presentation with generators $a_{i}, b_{i}$, and $c, i=1, \ldots, n$, and relations

$$
\begin{gathered}
a_{i}^{-1}=b_{i}^{q_{i}} \cdots b_{n}^{q_{n}} c^{k} b_{n}^{-q_{n}} \cdots b_{i}^{-q_{i}} c^{-k}, \\
b_{i}^{-1}=c^{-k} a_{i}^{-s_{i}} \cdots a_{1}^{-s_{1}} c^{k} a_{1}^{s_{1}} \cdots a_{i}^{s_{i}}, \\
c^{-h}=b_{n}^{-q_{n}} \cdots b_{1}^{-q_{1}} a_{1}^{s_{1}} b_{1}^{q_{1}} \cdots a_{n}^{s_{n}} b_{n}^{q_{n}} a_{n}^{-s_{n}} \cdots a_{1}^{-s_{1}} .
\end{gathered}
$$

This presentation is geometric; that is, it is induced by a genus $2 n+1$ Heegaard diagram of $K_{\alpha / \beta}(\gamma)$. We can eliminate the generator $b_{1}^{-1}=c^{-k} a_{1}^{-s_{1}} c^{k} a_{1}^{s_{1}}$ to get a balanced presentation of $\pi_{1}\left(K_{\alpha / \beta}(\gamma)\right)$ with $2 n$ generators. We see that the curve of the diagram represented by the relator $b_{1} c^{-k} a_{1}^{-s_{1}} c^{k} a_{1}^{s_{1}}$ has exactly one point in common with the curve (on the Heegaard surface) represented by the generator $b_{1}$. Then the pair of such curves determines a reducible handle in the diagram. Cancelling it yields a new Heegaard diagram of $K_{\alpha / \beta}(\gamma)$ (with 
genus $2 n$ ) inducing the above $2 n$-balanced presentation for $\pi_{1}\left(K_{\alpha / \beta}(\gamma)\right)$. The recurrence formulae of the statement are obtained as follows:

$$
\begin{aligned}
a_{i+1}^{-1} & =b_{i+1}^{q_{i+1}} \cdots b_{n}^{q_{n}} c^{k} b_{n}^{-q_{n}} \cdots b_{i+1}^{-q_{i+1}} c^{-k} \\
& =b_{i}^{-q_{i}} b_{i}^{q_{i}} b_{i+1}^{q_{i+1}} \cdots b_{n}^{q_{n}} c^{k} b_{n}^{-q_{n}} \cdots b_{i+1}^{-q_{i+1}} b_{i}^{-q_{i}} b_{i}^{q_{i}} c^{-k} \\
& =b_{i}^{-q_{i}} a_{i}^{-1} c^{k} b_{i}^{q_{i}} c^{-k}, \\
b_{i+1}^{-1} & =c^{-k} a_{i+1}^{-s_{i+1}} a_{i}^{-s_{i}} \cdots a_{1}^{-s_{1}} c^{k} a_{1}^{s_{1}} \cdots a_{i}^{s_{i}} a_{i+1}^{s_{i+1}} \\
& =c^{-k} a_{i+1}^{-s_{i+1}} c^{k} b_{i}^{-1} a_{i+1}^{s_{i+1}}
\end{aligned}
$$

for $i=1, \ldots, n-1$. Using these relations we can successively eliminate the generators $a_{i+1}$ and $b_{i+1}$ for $i=1, \ldots, n-1$ (together with $b_{1}=a_{1}^{-s_{1}} c^{-k} a_{1}^{s_{1}} c^{k}$ ). The Tietze moves on the obtained presentations for the group $\pi_{1}\left(K_{\alpha / \beta}(\gamma)\right)$ correspond geometrically to cancel reducible handles in the current Heegaard diagrams (of decreasing genus) inducing those presentations. So $K_{\alpha / \beta}(\gamma)$ can be represented by a Heegaard diagram of genus 2 . Such a diagram induces a geometric presentation for $\pi_{1}\left(K_{\alpha / \beta}(\gamma)\right)$ with two generators $a_{1}$ and $c$ and two relators obtained by applying the above recurrence algorithm. This shows that the genus of $K_{\alpha / \beta}(\gamma)$ is at most 2 . Now we claim that the genus is exactly 2 . This follows from the fact that 2-bridge knots have tunnel number equal to one and no lens space surgeries (see, e.g., [5]).

To complete the section we write explicitly the geometric presentations for $\pi_{1}\left(K_{\alpha / \beta}(\gamma)\right)$ with $\alpha / \beta=\left[-2 s_{1}\right.$, $\left.2 q_{1}, \ldots,-2 s_{n}, 2 q_{n}\right]$ for $n=1,2$.

Corollary 4. The fundamental group of the surgery manifold $K_{\alpha / \beta}(\gamma), \gamma=h / k$ and $\alpha / \beta=\left[-2 s_{1}, 2 q_{1}\right]=\left(-4 q_{1} s_{1}+1\right) /\left(2 q_{1}\right)$, has the geometric presentation:

$$
\begin{gathered}
\pi_{1}\left(K_{\alpha / \beta}(\gamma)\right)=\left\langle a_{1}, c: a_{1}\left[a_{1}^{-s_{1}}, c^{-k}\right]^{q_{1}}\left[a_{1}^{-s_{1}}, c^{k}\right]^{q_{1}}=1,\right. \\
\left.c^{h}\left[c^{-k}, a_{1}^{-s_{1}}\right]^{q_{1}}\left[c^{-k}, a_{1}^{s_{1}}\right]^{q_{1}}=1\right\rangle,
\end{gathered}
$$

where $[x, y]=x y x^{-1} y^{-1}$.

Corollary 5. The fundamental group of the surgery manifold $K_{\alpha / \beta}(\gamma), \gamma=h / k, \alpha / \beta=\left[-2 s_{1}, 2 q_{1},-2 s_{2}, 2 q_{2}\right]$, that is, $\alpha=$ $16 q_{1} q_{2} s_{1} s_{2}-4\left(q_{1} s_{1}+q_{2} s_{1}+q_{2} s_{2}\right)+1$ and $\beta=-8 q_{1} q_{2} s_{2}+$ $2\left(q_{1}+q_{2}\right)$, has the geometric presentation with generators $a_{1}$ and $c$ and relations $a_{2}\left[b_{2}^{q_{2}}, c^{k}\right]=1$ and

$$
c^{h} b_{2}^{-q_{2}} b_{1}^{-q_{1}} a_{1}^{s_{1}} b_{1}^{q_{1}} a_{2}^{s_{2}} b_{2}^{q_{2}} a_{2}^{-s_{2}} a_{1}^{-s_{1}}=1,
$$

where

$$
\begin{aligned}
b_{1}= & {\left[a_{1}^{-s_{1}}, c^{-k}\right], \quad a_{2}=\left[a_{1}^{-s_{1}}, c^{k}\right]^{q_{1}} a_{1}\left[a_{1}^{-s_{1}}, c^{-k}\right]^{q_{1}}, } \\
b_{2}= & \left(\left[a_{1}^{-s_{1}}, c^{k}\right]^{q_{1}} a_{1}\left[a_{1}^{-s_{1}}, c^{-k}\right]^{q_{1}}\right)^{-s_{2}}\left[a_{1}^{-s_{1}}, c^{-k}\right] c^{-k} \\
& \times\left(\left[a_{1}^{-s_{1}}, c^{k}\right]^{q_{1}} a_{1}\left[a_{1}^{-s_{1}}, c^{-k}\right]^{q_{1}}\right)^{s_{2}} c^{k} .
\end{aligned}
$$

From Theorem 3 and [5] we also have the following consequence (for $n=1$ see [6]).

Corollary 6. Let $K_{\alpha / \beta}$ be a hyperbolic 2-bridge knot, where $\alpha / \beta=\left[-2 s_{1}, 2 q_{1}, \ldots,-2 s_{n}, 2 q_{n}\right]$ and $n \geq 2$. Then the surgery manifolds $K_{\alpha / \beta}(\gamma), \gamma \neq \infty$, are hyperbolic and have Heegaard genus 2 . The volumes of such manifolds can be made arbitrarily large.

Proof. As done in [16, p. 725], for a slightly different link (see also [11, 17]), it follows that the links $\mathscr{L}_{2 n+1}$ are hyperbolic with volume approximately $(2 n-1)(7.32772 \ldots)$. Furthermore, $\mathscr{L}_{2 n+1}$ is amphicheiral and its symmetry group is isomorphic to $\mathbb{Z}_{2} \times D_{4}$, where $D_{4}$ is the dihedral group of order 8 . On choosing a framing for each unknotted component of $\mathscr{L}_{2 n+1}$, we can perform $1 / n$ Dehn surgery on each of the unknotted components of $\mathscr{L}_{2 n+1}$. This produces the hyperbolic 2-bridge knot $\mathbf{K}_{n}=K_{\alpha / \beta}$, where $\alpha / \beta=$ $[-2 n, 2 n, \ldots,-2 n, 2 n]$. Thurston's hyperbolic Dehn surgery theorem [3] in this context says that $\mathbf{K}_{m}$ has a $2 n$-long continued fraction consisting of $2 m$ 's with volumes of $\mathbb{S}^{3} \mid$ $\mathbf{K}_{m}$ converging to that of $\mathbb{S}^{3} \backslash \mathscr{L}_{2 n+1}$ as $m$ goes to infinity. Since these are getting arbitrarily large, the result follows. In fact, the volumes of the surgery hyperbolic manifolds $\mathbf{K}_{n}(\gamma)$, $\gamma \neq \infty$ and $n \geq 2$, become arbitrarily large as $n$ goes to infinity. The fact that the volumes of these manifolds can be arbitrarily large is also a consequence of work by Lackenby on volumes of hyperbolic alternating links (see [18]). (See, e.g., [19, 20] for interesting estimates of volumes for hyperbolic manifolds arising from right-angled Coxeter polyhedra.)

\section{Covering Properties}

In this section we study covering properties of our surgery manifolds. Using Montesinos' trick [8], we prove that such manifolds are 2-fold branched covers of a connected sum of lens spaces. Moreover, it follows that a very large subclass of our surgery manifolds are 2-fold coverings of the 3-sphere branched over well-specified clearly depicted links. Finally, we show explicitly what the branched cover looks like for the surgeries on a large class of links including 2-bridge knots as very particular case.

Theorem 7. Suppose that $r_{i}$ is odd for every $i=1, \ldots, n$. Then the surgery manifold $M_{n}\left(r_{i} / s_{i} ; p_{i} / q_{i} ; h / k\right)$ is 2-fold branched covering of the connected sum of $n$ lens spaces $L\left(r_{1}, 2 s_{1}\right) \# \cdots \# L\left(r_{n}, 2 s_{n}\right)$.

Proof. As shown in Figure 4(a), there is an orientationpreserving involution $\rho$ in $\mathbb{S}^{3}$ which induces an involution with two fixed points (resp., without fixed points) in each component $L_{0}$ and $K_{i}$ (resp., $L_{i}$ ) of $\mathscr{L}_{2 n+1}$, for $i=1, \ldots, n$. Here we will assume $n \geq 2$. For $n=1$ see [6]. Let $\mathscr{L}^{\prime}$ be the link consisting of those components of $\mathscr{L}_{2 n+1}$ for which the number of fixed points of $\rho$ is different from two. Let $p: \mathbb{S}^{3} \rightarrow \mathbb{S}^{3}$ be the 2-fold cyclic branched covering of the 3-sphere $\mathbb{S}^{3}$ defined by $\rho$. By Theorem 2 of [8] the manifold obtained by doing surgery on $\mathscr{L}_{2 n+1}$ is a 2 -fold 


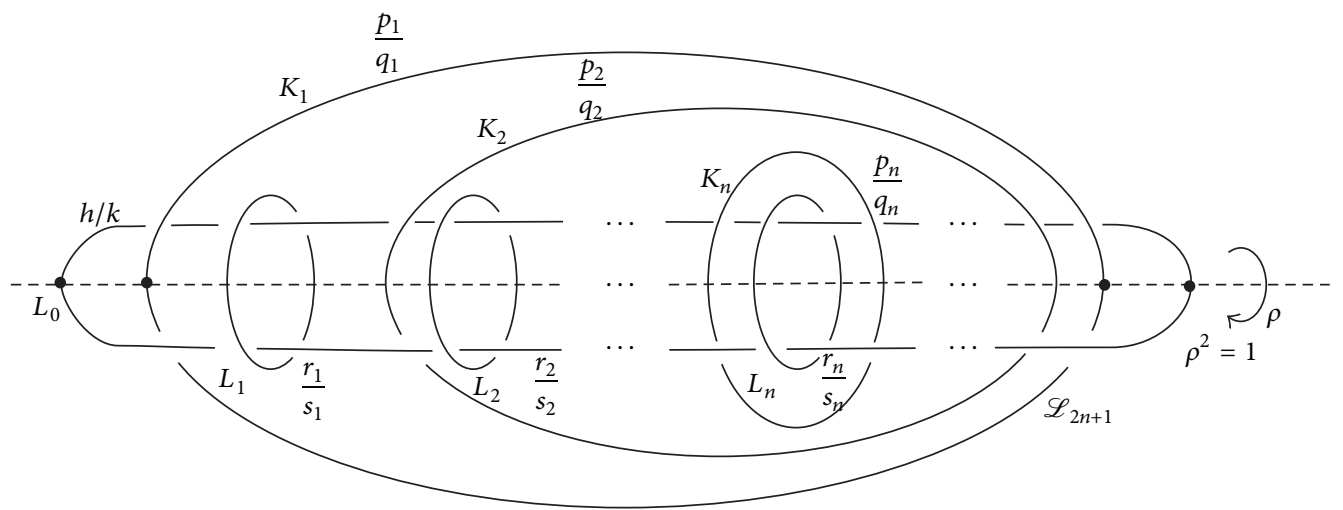

(a)

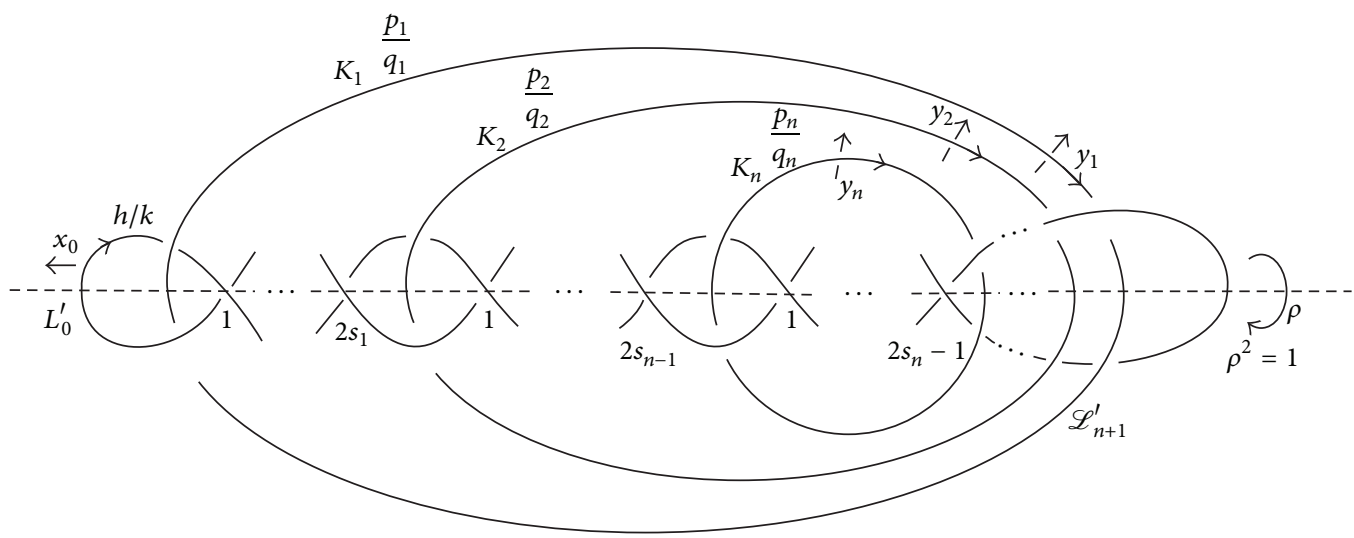

(b)

FIGURE 4: The 2 -symmetric planar projections of the links $\mathscr{L}_{2 n+1}$ and $\mathscr{L}_{n+1}^{\prime}=\mathscr{L}_{2 n+1}\left(1 / s_{1}, \ldots, 1 / s_{n}\right)$.

cyclic covering branched over a manifold obtained by doing surgery on $p\left(\mathscr{L}^{\prime}\right)$. But $p\left(\mathscr{L}^{\prime}\right)$ is a trivial link. Now the result follows from the fact that surgery on a trivial link produces a connected sum of lens spaces. This yields a representation of our surgery manifolds as branched coverings of a connected sum of lens spaces.

Let $\mathscr{L}^{\prime}{ }_{n+1}$ be the oriented link in $\mathbb{S}^{3}$ with $n+1$ components (which we denote by $L_{0}^{\prime}$ and $K_{i}$ for $i=1, \ldots, n$ ) obtained from $\mathscr{L}_{2 n+1}$ by doing $1 / s_{i}$ Dehn surgeries on its $L_{i}$ components, $i=$ $1, \ldots, n$. The link $\mathscr{L}_{n+1}^{\prime}$ is strongly invertible (see Figure 4(b)); that is, there is an orientation-preserving involution of $\mathbb{S}^{3}$, also denoted by $\rho$, which induces in each component of $\mathscr{L}_{n+1}^{\prime}$ an involution with two fixed points. We remark that in Figure 4(b) the last string has $2 s_{n}-1$ crossings instead of $2 s_{n}\left(s_{n} \geq 1\right)$ because we have shifted the subarc (at the final crossing) of the link from the bottom to the top. This permits losing a crossing. Now we recall the statement of Theorem 1 from [8]: let $M$ be a closed orientable 3-manifold that is obtained by doing surgery on a strongly-invertible link $L$ of $n$ components. Then $M$ is a 2 -fold cyclic covering of the 3 sphere branched over a link of at most $n+1$ components. Thus Theorem 1 of [8] applies to our case, and we can state that the manifolds $M_{n}\left(r_{i} / s_{i} ; p_{i} / q_{i} ; h / k\right)$ with $r_{i}=1, i=1, \ldots, n$, are 2-fold coverings of $\mathbb{S}^{3}$ branched over a link of at most $n+2$ components. Now we apply the Montesinos algorithm, given in [8], to describe explicitly the branch sets of the above 2fold branched coverings. Let $\mathscr{L}_{r}\left(p_{i} / q_{i} ; h / k\right)$, where $r=2 s_{1}+$ $\cdots+2 s_{n}$, denote the branch set of the 2 -fold branched covering $M_{n}\left(r_{i} / s_{i} ; p_{i} / q_{i} ; h / k\right)$, with $r_{i}=1$ for $i=1, \ldots, n$, of $\mathbb{S}^{3}$ which corresponds to the involution $\rho$ shown in Figure 4(b) (recall that $s_{i} \geq 1$ for $\left.i=1, \ldots, n\right)$. Let $\mathbf{m}_{i}=y_{i}$ be the meridians of the components $K_{i}$ of $\mathscr{L}_{n+1}^{\prime}$ and $\mathbf{m}_{0}=x_{0}$ the meridian of the component $L_{0}^{\prime}$ of $\mathscr{L}_{n+1}^{\prime}$. The pair $\left(\mathbf{m}_{i}, \boldsymbol{\ell}_{i}\right)$, where $\boldsymbol{\ell}_{i}$ is the longitude of $K_{i}$, is a preferred frame; that is, $\boldsymbol{\ell}_{i} \sim 0$ in the exterior space $\mathbb{S}^{3} \backslash K_{i}$ and $l k\left(\mathbf{m}_{i}, \boldsymbol{\ell}_{i}\right)=1$ for $i=1, \ldots, n$. The pair $\left(\mathbf{m}_{0}, \boldsymbol{\ell}_{0}^{\prime}\right)$, where $\boldsymbol{\ell}_{0}^{\prime}$ is the longitude of $L_{0}^{\prime}$, is not a preferred frame since $\boldsymbol{\ell}_{0}^{\prime} \sim-(r-1) \mathbf{m}_{0}$ in $\mathbb{S}^{3} \backslash L_{0}^{\prime}$, where $r=2 s_{1}+\cdots+2 s_{n}$. To have a preferred frame, we take the pair $\left(\mathbf{m}_{0}, \boldsymbol{\ell}_{0}\right)$, where $\boldsymbol{\ell}_{0}=\boldsymbol{\ell}_{0}^{\prime}+(r-1) \mathbf{m}_{0}$. Let $V$ be a regular neighbourhood of the link $\mathscr{L}_{n+1}^{\prime}$ in $\mathbb{S}^{3}$. Without loss of generality, we can choose $V$, the meridians $\mathbf{m}_{i}$, and the longitudes $\boldsymbol{\ell}_{i}, i=0,1, \ldots, n$, to be invariant under the involution $\rho$. The quotient space of $\mathbb{S}^{3}$ under $\rho$ is illustrated in Figure 5. The image of $V$ under the projection $\pi: \mathbb{S}^{3} \rightarrow \mathbb{S}^{3} / \rho$ consists of $n+1$ disjoint 3 -balls; $B_{0}, B_{1}, \ldots, B_{n}$, say. To obtain the branch set $\mathscr{L}_{r}\left(p_{i} / q_{i} ; h / k\right)$, where $r=2 s_{1}+\cdots+2 s_{n}$, via the Montesinos algorithm, we isotopy the $B_{i}$ 's along the images $\pi\left(\boldsymbol{\ell}_{i}\right)$ of the longitudes $\boldsymbol{\ell}_{i}$ and replace them by an $h / k$ rational tangle for $i=0$ and by $p_{i} / q_{i}$ rational tangles, for $i=1, \ldots, n$, as in Figure 6 .

Summarizing, we have proven the following main result. 


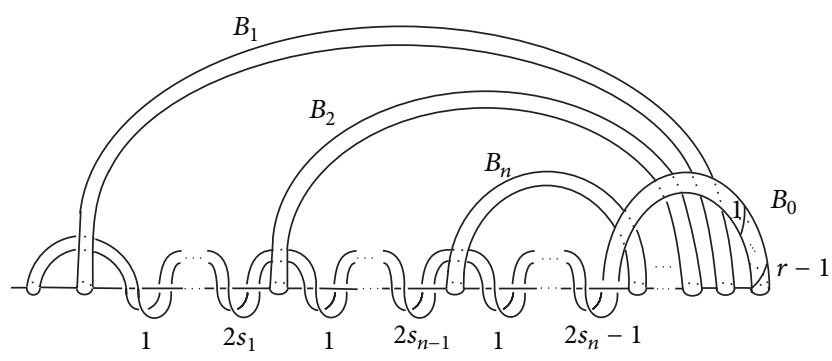

FIgURE 5: The quotient $\left(\mathbb{S}^{3} \backslash \operatorname{int} V\right) / \rho$.

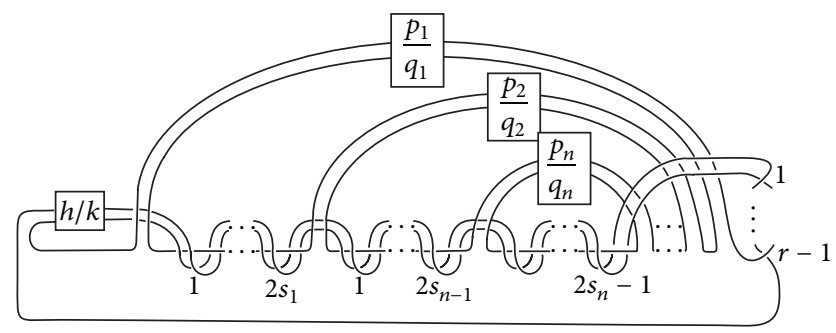

FIgURE 6: The link $\mathscr{L}_{r}\left(p_{i} / q_{i} ; h / k\right), r=2 s_{1}+\cdots+2 s_{n}$.

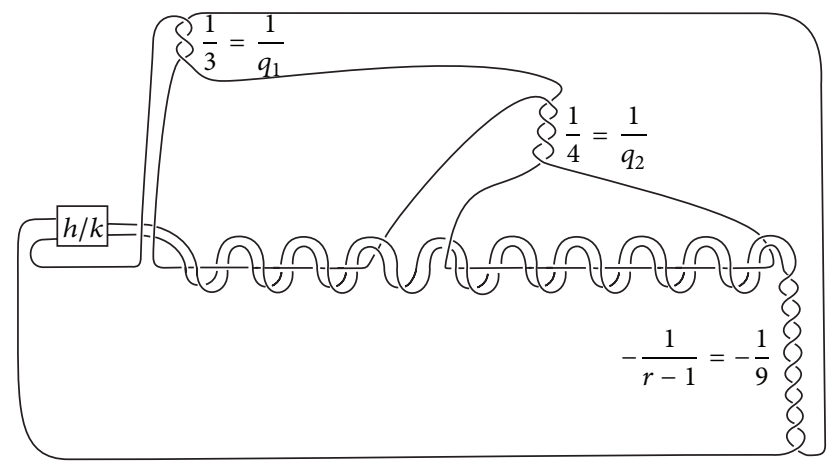

FIgURE 7 : The link $\mathscr{L}_{10}(1 / 3,1 / 4 ; h / k)$.

Theorem 8. Let $\mathscr{M}=M_{n}\left(r_{i} / s_{i} ; p_{i} / q_{i} ; h / k\right), r_{i}=1$ and $s_{i} \geq 1$, for $i=1, \ldots, n$, be the closed connected orientable 3-manifold obtained by Dehn surgeries on the components of the link $\mathscr{L}_{2 n+1}$. Then $\mathscr{M}$ is the 2-fold covering of the 3-sphere branched over the link $\mathscr{L}_{r}\left(p_{i} / q_{i} ; h / k\right)$, where $r=2 s_{1}+\cdots+2 s_{n}$, pictured in Figure 6.

Theorem 9. Let $K_{\alpha / \beta}(\gamma), \gamma=h / k \neq \infty$, be the closed connected orientable 3-manifold obtained by $\gamma$ Dehn surgery on the hyperbolic 2-bridge knot $K_{\alpha / \beta}$, where $\alpha / \beta=$ $\left[-2 s_{1}, 2 q_{1}, \ldots,-2 s_{n}, 2 q_{n}\right]$. Then $K_{\alpha / \beta}(\gamma)$ is the 2 -fold covering of the 3-sphere branched over the link $\mathscr{L}_{r}\left(p_{i} / q_{i} ; h / k\right)$, where $r=2 s_{1}+\cdots+2 s_{n}$ and $p_{i}=1$ for every $i=1, \ldots, n$.

For example, $K_{\alpha / \beta}(\gamma), \gamma=h / k \neq \infty$, where $\alpha / \beta=$ $[-4,6,-6,8]$, hence $\alpha=1049$, and $\beta=-274$, is the 2 fold covering of the 3 -sphere $\mathbb{S}^{3}$ branched over the link $L_{10}(1 / 3,1 / 4 ; h / k)$ as shown in Figure 7.

\section{Acknowledgments}

This work is performed under the auspices of the GNSAGA of the National Research Council (CNR) of Italy and partially supported by the Ministero dell'Istruzione, dell'Universitá e della Ricerca Scientifica (MIUR) of Italy.

\section{References}

[1] W. B. R. Lickorish, "A representation of orientable combinatorial 3-manifolds," Annals of Mathematics. Second Series, vol. 76, pp. 531-540, 1962.

[2] A. H. Wallace, "Modifications and cobounding manifolds," Canadian Journal of Mathematics, vol. 12, pp. 503-528, 1960.

[3] W. P. Thurston, The Geometry and Topology of 3-Manifolds, Lecture Notes, Princeton University Press, Princeton, NJ, USA, 1980.

[4] D. Rolfsen, Knots and Links, vol. 7 of Mathematics Lecture Series, Publish or Perish, Berkeley, Calif, USA, 1978.

[5] M. Brittenham and Y. Q. Wu, "The classification of exceptional Dehn surgeries on 2-bridge knots," Communications in Analysis and Geometry, vol. 9, no. 1, pp. 97-113, 2001.

[6] A. Cavicchioli, F. Spaggiari, and A. I. Telloni, "Dehn surgeries on some classical links," Proceedings of the Edinburgh Mathematical Society, vol. 54, no. 1, pp. 33-45, 2011.

[7] S. H. Kim and Y. Kim, "On hyperbolic 3-manifolds obtained by Dehn surgery on links," International Journal of Mathematics and Mathematical Sciences, vol. 2010, Article ID 573403, 8 pages, 2010.

[8] J. M. Montesinos, "Surgery on links and double branched covers of $\mathbb{S}^{3}$," in Knots, Groups and 3-Manifolds, L. P. Neuwirth, Ed., vol. 84 of Annals of Mathematics Studies, pp. 227-259, Princeton University Press, Princeton, NJ, USA, 1974.

[9] L. Moser, "Elementary surgery along a torus knot," Pacific Journal of Mathematics, vol. 38, pp. 737-745, 1971.

[10] M. Ochiai, “Dehn's surgery along 2-bridge knots," Yokohama Mathematical Journal, vol. 26, no. 1, pp. 69-75, 1978.

[11] C. Adams, "Hyperbolic knots," Geometry \& Topology, http:// arxiv.org/abs/math/0309466.

[12] R. P. Osborne and R. S. Stevens, "Group presentations corresponding to spines of 3-manifolds. I," American Journal of Mathematics, vol. 96, pp. 454-471, 1974.

[13] R. P. Osborne and R. S. Stevens, "Group presentations corresponding to spines of 3-manifolds. II, III," Transactions of the American Mathematical Society, vol. 234, no. 1, pp. 213-243, 245-251, 1977.

[14] J. H. Conway, "An enumeration of knots and links, and some of their algebraic properties," in Computational Problems in Abstract Algebra, J. Leech, Ed., pp. 329-358, Pergamon Press, Oxford, UK, 1969.

[15] A. Kawauchi, A Survey of Knot Theory, Birkhäuser, Basel, Switzerland, 1996.

[16] P. J. Callahan and A. W. Reid, "Hyperbolic structures on knot complements," Chaos, Solitons and Fractals, vol. 9, no. 4-5, pp. 705-738, 1998, Knot theory and its applications.

[17] C. Adams, Hyperbolic structures on link complements [Ph.D. thesis], University of Wisconsin, Madison, Wis, USA, 1983.

[18] M. Lackenby, "The volume of hyperbolic alternating link complements," Proceedings of the London Mathematical Society, vol. 88, no. 3, pp. 204-224, 2004. 
[19] A. Yu. Vesnin and D. Repovs, "Two-sided bounds for the volumes of right-angled hyperbolic polyhedra," Rossiǔskaya Akademiya Nauk. Matematicheskie Zametki, vol. 89, no. 1, pp. 12-18, 2011.

[20] D. Repovs and A. Vesnin, Normalized Volumes of Right-Angled Hyperbolic Polyhedra, vol. 279 of Research Preprint Series, Abdus Salam School of Mathematical Sciences, Lahore, Pakistan, 2011. 


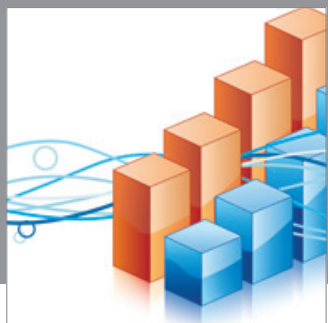

Advances in

Operations Research

mansans

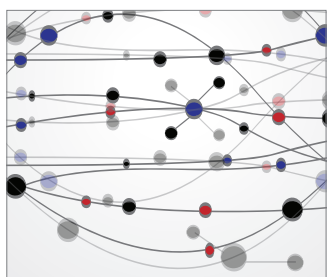

The Scientific World Journal
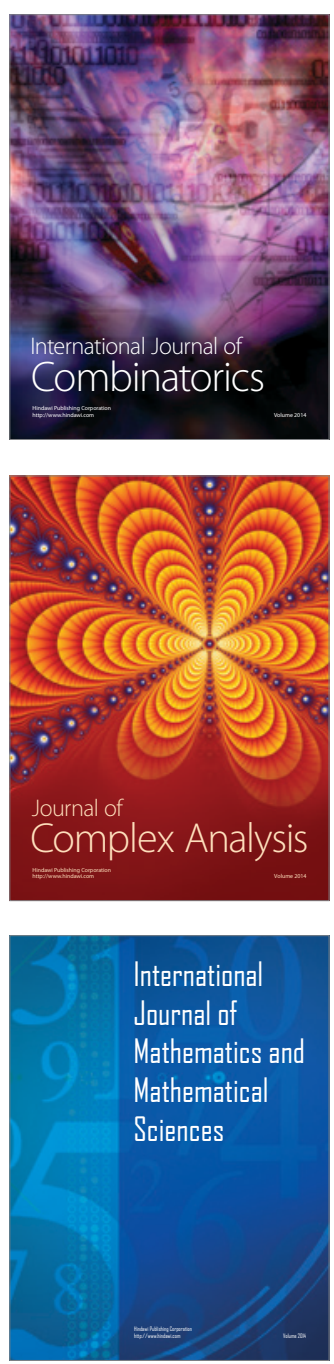
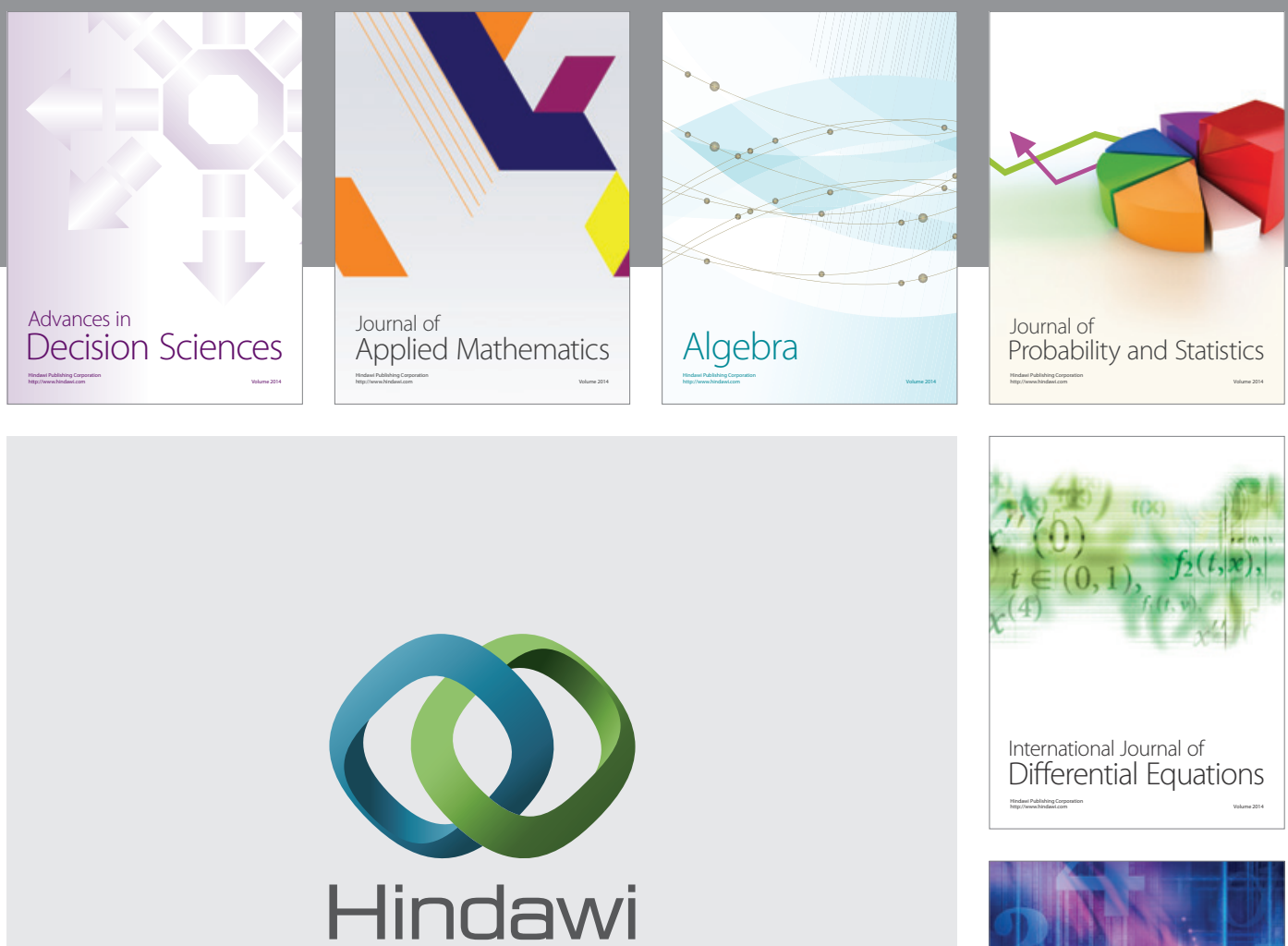

Submit your manuscripts at http://www.hindawi.com
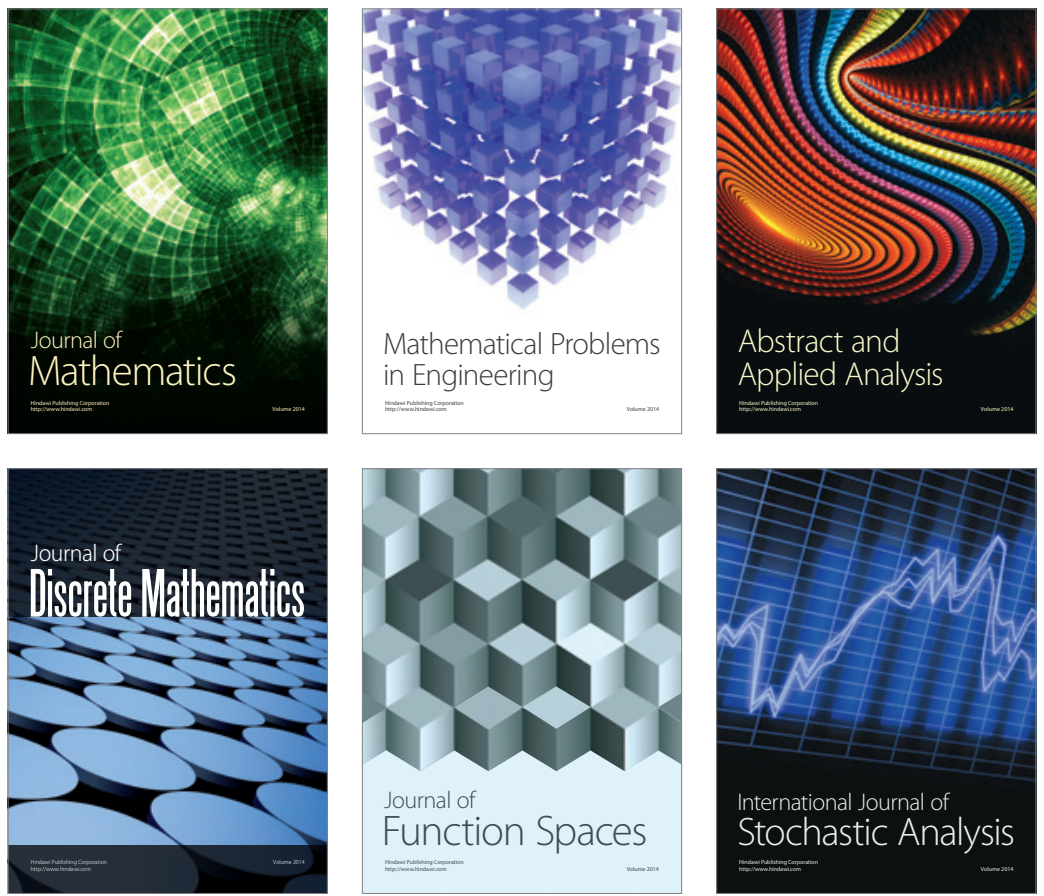

Journal of

Function Spaces

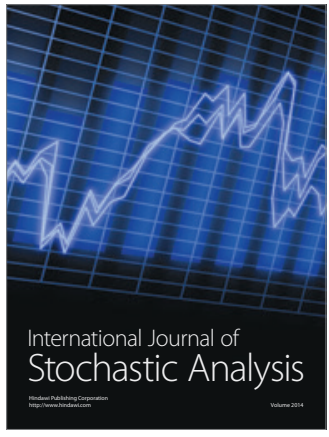

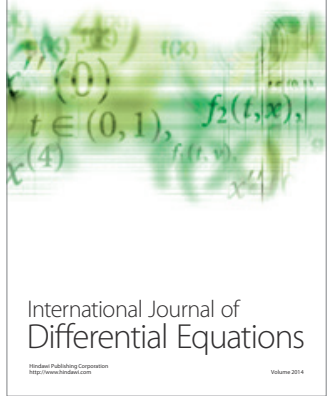
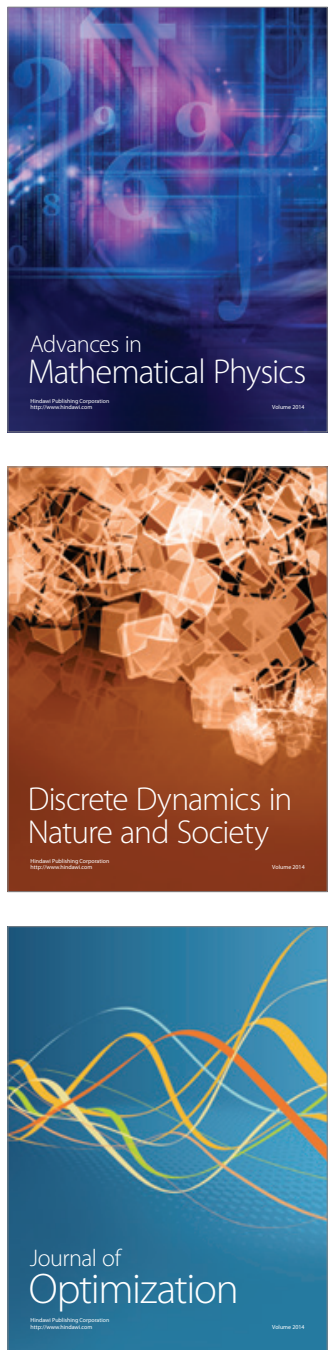